\title{
FACING THE CHALLENGE: DO WE HAVE AN INTEGRATION IDEA?
}

\section{Vladislav Medintsev}

Laboratory of methodology and theory of psychology, G.S. Kostiuk Institute of Psychology of the National Academy of Educational Sciences of Ukraine

\author{
Correspondence: \\ Vladislav Medintsev \\ vladislav-medintsev@yandex.ru
}

For several decades, psychologists have been expressing concern about the growing theoretical and methodological disunity of psychological knowledge. To counteract this trend, various integrative approaches have been put forward. I suggest that for the successful development of integrative research, psychologists must come to an agreement on the general, conceptual and methodological ideas of integration. The selection of the most optimal integration ideas can be carried out by comparing them in terms of simplicity, powerfulness and potential to become unifying. The article also suggests distinguishing between two types of integration ideas: for integrating existing psychological knowledge (the idea of descriptive integration) and for conducting new research (the idea of prescriptive integration). The proposals put forward, I hope, will serve to deepen the understanding of the methodological perspectives for integrative approaches in psychology.

Keywords: idea, theory, fragmentation, integration, methodology, simplicity, powerfulness.

Psychologists, concerned about the growing fragmentation of their science, are searching how to overcome it through the psychological knowledge unification. The earliest publication on this topic that I found dates back to 1970 (Kendler, 1970), it outlines the problems in psychological science that are caused by its fragmentation, and emphasizes that the achievement of psychological knowledge unification essentially depends on the adoption of common criteria for explanation. In 1986, the Society for Unification Psychology was created and its goals were proclaimed (Society, 1986). It seems not possible to find traces of this society further activities, but the goals defined by its founders - to stimulate discussion of issues of unity and disunity in psychology - can be considered achieved to a certain extent. Over time, the publications on this issue, along with the term "unification", the term "integration" began to be used. (Baucal and Krstić, 2020; Cleeremans, 2010; Holbrook, 2013). And the development of approaches to the psychological knowledge integration is becoming increasingly important. At the same time, discussions continue about the need and possibility of transformations in psychology, including integration. Opponents of integration, as a rule, accentuate the widespread position, according to which psychology is a science with its own theoretical and methodological means, historically established theoretical and empirical forms and traditions of acquiring new knowledge - it will remain so in the future (Gijsbers, 2007, Mironenko and Sorokin, 2020). However, it was the traditions and forms of scientific psychological knowledge existing for a long time that gave rise to its modern disunity and the problems associated with it, including those concerning the scientific status of psychology (Lucas and Meike, 2016) and the actively discussed "replication crisis".

In the 1980s, the idea of a new philosophy was put forward by Staats A.W. (1987), who suggested it as the basis for solving the main problems caused by disunity. In particular, it will be possible to put forward theories of a new type, which are necessary to transform theoretical chaos into systematic, common sense and unified knowledge. This philosophy opens up a new field of research, a unified psychology, aimed at examining various aspects of disunity and the possibility of unification. 
The issue of integration is essentially related to the improvement of the psychology theoretical bases. In one of the recent publications on this topic Borghi and Fini (2019) stressed that in psychology, theoretical and methodological issues are not given sufficient attention, in particular by scientific editorials. Within the "limits" designated by the authors, they represent the vision of altering the situation. It is difficult to disagree with their arguments about the need for an interdisciplinary dialogue, the emphasis of theoretical foundations in the ongoing research and the epistemological awareness of researchers. However, interdisciplinary dialogues have been taking place for a long time, a lot has also been written about the importance of theory in any scientific field, but disintegrative tendencies in psychology not only persist, but also deepen. The authors note: "empirically-informed philosophy can play a crucial integrative role, helping to build a more comprehensive view of the field and identifying links that cross disciplinary boundaries" (p. 1). What has been said would be achievable if philosophy itself were unified (see, for instance, on the modern psychology philosophical problems Edelman, 2012). Therefore, modern philosophy can be viewed not so much as an integrating discipline, but as one of the sources of difficulties in integrating psychological knowledge.

Matthews G. (2020) suggests that interdisciplinary communication can also be considered as a type of integration and may be provided as: competition, cooperation, coexistence. In another version interdisciplinary communication, consensus, incommensurability and ingenuity are emphasized (Holbrook, 2013). In the current debates on the prospects for the development of psychology, the integrative perspective is one of the considered, all the proposed options for perspectives certainly also deserve attention (see an overview of the recent discussion on the topic Getting Psychology onto its feet again Valsiner, 2020). It seems hard to disagree with the fact that "For psychology to become a real science - among its peers of chemistry and biology-it needs to move from its dominance on inductive inference to that of abductive discovery of how psychological worlds are organized" (p. 518). However, the methodology for such a transition has not yet been accepted by the psychological community, although there are constructive proposals (for example, Baucal and Krstić, 2020, Cleeremans, 2010, Yanchuk, 2018).

\section{SIMPLE, NEW, POWERFUL, UNIFYING}

All of the above and many other judgments concerning the aspects of the psychological knowledge integration reflect its fragmentation, that is, integration approaches are also conceptually and methodologically heterogeneous. So, it makes sense to start with a search for the clearest general integration idea, and for this purpose one can turn to the philosophy of science. The first requirement for the growth of scientific knowledge, which was put forward by K. Popper, was: "The new theory should proceed from some simple, new, and powerful, unifying idea about some connection or relation (such as gravitational attraction) between hitherto unconnected things (such as planets and apples) or facts (such as inertial and gravitational mass) or new 'theoretical entities' (such as field and particles)" (Popper, 1963, p. 241). He added that the requirement for simplicity is vague and difficult to state clearly and stressed the need to relativize the comparison of simplicity in relation only to those hypotheses that compete with each other as solutions to a particular problem or set of problems. I suggest that solving such a large-scale task as the psychological knowledge integration requires a simple, new, powerful and unifying idea.

Even the author of one of the most difficult scientific theories to understand, A. Einstein, emphasized the need to achieve the goals of science "by applying a minimum of primary concepts and relationships (achieving, as far as possible, logical unity in the picture of the world, that is, to a minimum of logical elements)" (translated from Einstein, 1936, p. 317). In scientific knowledge, there are examples of clear simplicity criteria (for natural numbers in mathematics like), but there is still no general approach to assessing the simplicity of theories and hypotheses. There is a widespread belief among psychologists in the immeasurably higher complexity of the human psyche compared to the subject of any natural science discipline, which indirectly justify logically "vague" psychological theories. Despite this, many psychological theories, which at different times "conquered" the minds of researchers, were quite simple in their main provisions. Thus, not 
only in natural science, but also in psychology "the strength of the theory lies in its inner consistency and the simplicity of its fundamental assumptions" (Einstein and Infeld, 1938, p. 260).

The novelty of any idea and any cultural form is relative, which is reflected in the aphorisms such as "Everything old is new again" and "No man ever steps in the same river twice". There are many prominent examples of the old ideas "reincarnation" due to the enrichment of their content in new cultural realities. Consequently, it is not so much the absolute novelty of a scientific idea that is important as its novelty with a respect to a number of ideas that have already advanced to solve a particular scientific problem. The powerfulness of an idea can be assessed when and if a methodology for research and understanding of a known phenomenology will be developed on its basis. And this methodology will be considered more rational, corresponding to knowledge and practice in other fields of science for conceptualizing new phenomenology or/and for predicting new phenomena. That is, the powerfulness of an idea can only be assessed over time.

Unifying ideas are those that are shared by groups of people. Such ideas have been known for a long time - historians, philosophers and cultural scientists have written a lot about them, analyzing processes in social and another types of groups. Unifying ideas can be localized within small social groups, in particular, adherents of a certain scientific school or co-researchers. The problem of the relationship between personality psychology and social psychology - competition, cooperation, and coexistence - discussed in Matthews, 2020, is also linked to unifying ideas. In the modern science in each specific case, in a certain combination, all three types of relations are realized. These types also characterize relationships between groups of researchers, which may differ in their positions on the study of the psyche, but for cooperation they need unifying ideas. It seems clear that the principle of coexistence is contrary to any form of integration, so unity in psychology can only be achieved through cooperation and competition, which requires unifying ideas.

\section{THE IDEA OF INTEGRATION}

In integrative theoretical studies, one can single out statements that define approaches to understanding the essence of integration in psychology. According to the studies discussed above: (a) psychology should become a discipline in which a person can be understood in the synthesis of knowledge obtained in various psychological and non-psychological disciplines; (b) the psychological knowledge integration should be about its transformation on the basis of a new philosophy; (c) the psychological knowledge integration requires a common integrative conceptual framework; (d) the psychological knowledge integration is only possible on the basis of unified explanation criteria.

Summarizing these statements, one can formulate the idea of integration in psychology (or in human studies), for example, as follows: establishing a connection between all known components of psychological knowledge (or more broadly, human studies components). Such an idea can be regarded as a general integration idea - it seems simple, to a certain extent new and can become unifying for the integration supporters (however, it is difficult to assess its powerfulness). Whatever the general integration idea, it can be conceptualized in various ways, and the above statements can be considered as conceptual integration ideas. Conceptual integration ideas are different in their theoretical bases, they express the supposed forms of conceptualizing the connections between the components of the existing psychological, or a broader body of knowledge. None of them has yet become unifying, a comparison of their simplicity and powerfulness is hardly possible, since researchers focus on arguing their approaches rather than elaborating the methodological frameworks. Each conceptual idea of integration, in turn, can be methodologically implemented in different ways. In this regard, in addition to the basic and conceptual ones, it makes sense to consider the methodological integration ideas. Some integration approaches contain a more or less detailed methodological integration ideas. For example, if above suggestion (c) is considered as a conceptual integration idea, then its methodological realizations can be a three-dimensional matrix for structuring the associated 
results of psychology (Cleeremans, 2010) or the four-dimensional continuum of psychological phenomenology interdetermination (Yanchuk, 2018).

Popper's claims for new theories seem to be interconnected: only a simple, in comparison with others, idea, recognized by the scientific community as methodologically powerful, can become unifying one. The novelty of an idea, as noted above, hardly matters when solving integration problems. None idea put forward to date on the psychological knowledge integration has become unifying, there is no organized discussion in which to compare their simplicity and powerfullness. In addition, integrative research has an important methodological feature. It is known that any scientific idea must be concretized in theory and then tested empirically. But testing integration ideas is hardly possible in the near future. Thus, the comparison of integration ideas requires the development of strict criteria - this seems to be the only methodological route to choose the most promising among them.

Integration studies are concerned with both past and future psychological knowledge. But one can doubt the possibility of creating a unified theoretical means to integrate the results of completed studies and, on this basis, develop the methods for future research. This is evidenced by the experience of creating both general psychological theories and theories in psychological disciplines, as well as the fact that integration studies in psychological science have been going on for several decades without achieving the expected results. The theoretical foundations of completed studies, as well as the philosophical and even ideological attitudes of their authors, are so different that they can hardly be integrated into a clear methodological framework that would become an effective means for new research. In this regard, it makes sense to consider two components of the general integration idea: the descriptive integration idea and the prescriptive integration idea. The first can be implemented in theory, to describe the relationship between all known components of psychological knowledge. The second should be implemented in a unified methodological means for the new research. Of course, this tool should make it possible to apply the theoretical and methodological knowledge accumulated in psychology. I assume that formalized conceptual means are indispensable for implementing the idea of prescriptive integration. I believe that on the basis of formalized means using AI and IT, it is possible to build the necessary clear theoretical basis for creating complex multilevel theoretical frameworks for studying the human psyche and the patterns of its changes.

\section{CONCLUSION}

For several decades, integrative problems have been the subject of discussion among psychologists, but solving these problems has not yet become part of the psychological mainstream. Nevertheless, there is a growing number of scholars who regard integration as the only prospect for strengthening the status of psychology as a full-fledged science and significantly improving the effectiveness and scientific credibility of psychological research. Given the historical fragmentation of our science and its avalanche-like growth, the development and implementation of the integration project can be confidently attributed to the challenges we face. To meet this challenge as effectively as possible, integrative research needs to be significantly improved, since they have inherited a tendency towards fragmentation from psychological tradition. So, many theoretical and methodological integration solutions can be formulated, and the problem of "integrating integrative approaches" may become relevant in the near future. One of the possible responses to this challenge is to define a general integration idea, then conduct a comparative analysis of existing conceptual and methodological ideas on their simplicity, powerfulness and potential to become unifying for the entire psychological community.

\section{REFERENCES}

Baucal, A. and Krstić, K. (2020). Searching for an Integrative Theoretical Framework for Psychology: Evolutionary Psychology is Needed, But Not Sufficient. Integr. psych. behav. 54, 579-588. doi: 10.1007/s12124-020-09551-2 
Borghi, A. M, and Fini C. (2019). Theories and Explanations in Psychology. Front. Psychol. 2019.10: 958. doi: 10.3389/fpsyg.2019.00958.

Cleeremans, A. (2010). The grand challenge for psychology: integrate and fire! Front. Psychol, 22 April 2010. doi: 10.3389/fpsyg.2010.00012

Edelman, S. (2012). Six challenges to theoretical and philosophical psychology. Front. Psychol. 2012.3: 219. doi: 10.3389/fpsyg.2012.00219.

Einstein, A. (1936), Physik und Realität. Journ. Franklin Institut, 1936, Vol. 221, \# 3, P. 313 347.

Einstein, A. and Infeld L. (1938). The evolution of physics. Cambridge University Press, London, 1938.

Gijsbers, V. (2007). Why unification is neither necessary nor sufficient for explanation. Philosophy of Science 74 (4): 481-500. doi: 10.1086/524420

Holbrook, J. B. (2013). What is interdisciplinary communication? Reflections on the very idea of disciplinary integration. Synthese 190, 1865-1879. doi: 10.1007/s11229-012-0179-7

Kendler, H. H. (1970). The unity of psychology. Canadian Psychologist. Psychologie canadienne, 11 (1), 30-47. doi: 10.1037/h0082557

Lucas, B. M. and Meike, W. (2016). Debates about the Scientific Status of Psychology: Looking at the Bright Side. Integr Psych Behav 50:555-567. P. 555-567. doi: 10.1007/s12124-016-9352-8

Matthews, G. (2020). A Grand Challenge for Personality and Social Psychology: Competition, Cooperation, or Co-existence? Front. Psychol. 11: 1570. doi: 10.3389/fpsyg.2020.01570.

Mironenko, I. A. and Sorokin, P. S. (2020). Concerning Paradigmatic Status of Psychological Science: For a Flexible and Flowing Psychology in the Face of Practical and Theoretical Challenges. Integr. psych. behav. 54, 604-612. doi: 10.1007/s12124-020-09530-7

Popper, K.R. (1963). Conjectures and Refutations: The Growth of Scientific Knowledge. Routledge, London, 1963.414 P.

Society for Unification Psychology formed (1986). Theoretical \& Philosophical Psychology, 6 (1), 70-71. doi: 10.1037/h0091413

Staats, A. W. (1987). Unified Positivism Philosophy for the Revolution to Unity. Annals of Theoretical Psychology, vol 5. Springer, Boston, MA. doi: 10.1007/978-1-4615-6456-0_2

Valsiner, J. (2020). From Clay Feet to New Psychology: Starting the Move. Integr. psych. behav. 54, 515-520. doi: 10.1007/s12124-020-09564-X

Yanchuk, V. A. (2018). The Theoretical and Empirical Foundations of the SocioculturalInterdeterminist Dialogical Metatheory of the Integration of Psychological Knowledge, Journal of Russian \& East European Psychology, 55: 2-3, 241-286, doi: 10.1080/10610405.2018.1529531

Conflict of Interest: The author declare that the research was conducted in the absence of any commercial or financial relationships that could be construed as a potential conflict of interest.

Authors contribution statement: The author confirms being the sole contributor of this work and has approved it for publication. 